\title{
How much variation in referral rates among general practitioners is due to chance?
}

\author{
A T Moore, M O Roland
}

\begin{abstract}
A 20-fold variation in referral rates from general practitioners to hospital outpatient departments has been shown in studies published to date. Most of the hypotheses proposed to account for this variation have not been supported by these studies. A simple model was constructed, which showed that a significant part of the variation may be due to the fairly small numbers of referrals in most studies. Real differences may have been swamped by random variations in the small numbers of referrals. The statistical power of the studies may not have been high enough to determine which factors were significant in describing the variation and how much of the variation was due to differing clinical practice. There remains a substantial part of the variation that cannot be accounted for.
\end{abstract}

\section{Introduction}

Reports have shown a wide variation in the rate at

General Practice

Performance Review Unit, East Anglian Committee for Postgraduate Medical and Dental Education, Cambridge University School of Clinical Medicine, Addenbrooke's Hospital, Cambridge CB2 2QQ

A T Moore, MFCM, specialist in community medicine

M O Roland, MRCGP, director of studies in general practice

Correspondence to: $\mathrm{Dr}$ Moore.

BrMed J 1989;298:500-2 which individual general practitioners refer patients to hospital, ${ }^{1.3}$ indeed, in the recent government white paper on the review of the NHS the variation is said to be 20 -fold at the extremes. ${ }^{4}$ No one, however, has examined the role that random fluctuations arising by chance may have on the observed rates of referral (although the observation that many studies are based on small numbers of referrals has been made ${ }^{2}$ ). Many factors may influence rates of referral, including distance from the local hospitals, availability of public transport, social expectations, quality and quantity of the hospital service available, and the training, interests, and experience of the general practitioner. These factors are additional to the impact that variations in the morbidity of the population due to environmental and other factors and the age and sex structure of the population have on referral rates.

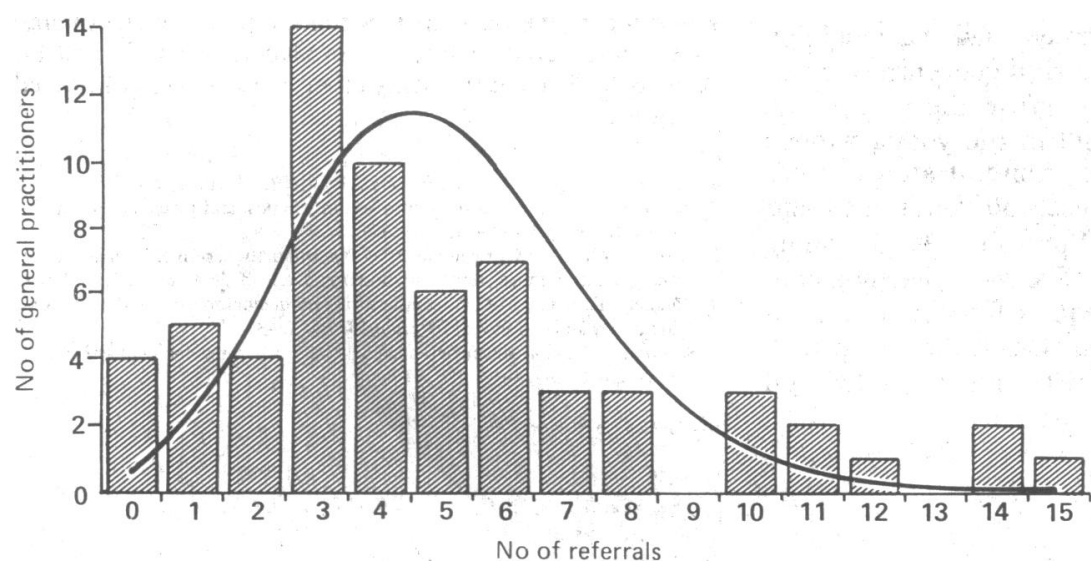

Theoretical model based on Poisson distribution showing wide range of referral rates from general practitioners to outpatient departments predicted on basis of chance alone. Histogram shows actual variation in numbers referred in study by Dowie, ${ }^{10}$ solid line gives numbers predicted by model
The fact that studies have frequently failed to show expected causes for variation may be due in part to a failure to take account of "random variation" in the numbers referred and so have insufficient statistical power. Referral is a fairly rare event and as such the number of referrals in a given period might be subject to considerable random fluctuation over and above the other factors - for example, in one month the average general practitioner may expect to refer about 28 people to an outpatient department. Some months this same doctor will refer more and other months less. This natural or random variation will result in the doctor having a higher than average referral rate one month and lower than average rate in other months, even if his or her referral behaviour with given clinical problems remains identical. Any systematic difference in referral rates will be superimposed on top of this "noise." If the random fluctuation is too large in comparison with the systematic differences then a study is unlikely to have the statistical power to show those differences.

The random fluctuation in numbers referred is particularly important if information on referral rates is used to help general practitioners examine their own referral behaviour. If attention were to be focused on the doctors who were at the highest and lowest ends of a distribution of referral rates and the ranks were due, in the most part, to the "noise" then the educational, and thus the patient, benefits would soon be undermined. A doctor could well be around the top centile one month and the bottom centile the next, not a situation likely to help critical self audit or to encourage the more reluctant to join a system of peer review.

Undoubtedly there are considerable financial implications inherent in a wide range of referral rates. ${ }^{5}$ In 1987 the government signalled its intention to provide general practitioners with their rates of referral. ${ }^{1}$ A crucial part of the presentation of referral rates is establishing some form of confidence interval so that high or low referral rates that arise by chance may be distinguished from systematically high or low rates.
Methods
If certain assumptions are made a crude theoretical model of the referral process can be constructed to examine the effect that random variation in the numbers referred may have on referral rates. The Poisson distribution was developed to describe fairly uncommon events occurring randomly in time. ${ }^{6}$ If we assume that conditions requiring referral (by some set of objective criteria) (a) arise independently of one another in the population, $(b)$ occur randomly in time, and $(c)$ are fairly rare events for an individual patient, then the Poisson distribution can be used to describe the chance that any given number will arise in a known population over a known time. If we further assume that the people with these conditions present them- 
TABLE I-Rates of referral and mean expected numbers (95\% confidence intervals) for different specialties for new outpatients (1986 figures)

\begin{tabular}{|c|c|c|c|c|}
\hline \multirow[b]{2}{*}{ Specialty } & \multirow{2}{*}{$\begin{array}{l}\text { Referrals/ } \\
10000 / \text { year }\end{array}$} & \multicolumn{3}{|c|}{ No expected ( $95 \%$ confidence interval) } \\
\hline & & per 1000 & per 2000 & per 10000 \\
\hline General surgery & 209 & $21(11$ to 30$)$ & $42(29$ to 50$)$ & 209 (180 to 238$)$ \\
\hline Trauma and orthopaedics & 201 & 20 (11 to 29 ) & $40(27$ to 53$)$ & 201 (173 to 229$)$ \\
\hline Ear, nose, and throat & 144 & 14 (6 to 22 ) & $29(18$ to 40$)$ & 144 (120 to 168$)$ \\
\hline Gynaecology & 137 & 14 (6 to 21$)$ & 27 (17 to 38$)$ & $137(114$ to 160$)$ \\
\hline Ophthalmology & 130 & $13(5$ to 21$)$ & $26(16$ to 36$)$ & $130(107$ to 153$)$ \\
\hline Obstetrics & 125 & $13(5$ to 20$)$ & $25(15$ to 35$)$ & $125(103$ to 147$)$ \\
\hline General medicine & 95 & $10(3$ to 16$)$ & 19 (10 to 28$)$ & 95 (75 to 115$)$ \\
\hline Dermatology & 85 & 9 (2 to 15$)$ & 17 (8 to 26$)$ & 85 (66 to 104$)$ \\
\hline Genitourinary medicine & 80 & 8 (2 to 14$)$ & 16 (8 to 24$)$ & 80 (62 to 98$)$ \\
\hline Oral surgery & 61 & 6 (1 to 11$)$ & $12(5$ to 19$)$ & $61(45$ to 77$)$ \\
\hline Rheumatology & 50 & $5(0$ to 10$)$ & 10 (3 to 17$)$ & 50 (36 to 64$)$ \\
\hline Urology & 33 & $3(0$ to 7$)$ & 7 (1 to 12$)$ & 33 (21 to 45$)$ \\
\hline Paediatrics & 32 & 3 (0 to 7$)$ & 6 (1 to 12 ) & 32 (20 to 44$)$ \\
\hline Radiotherapy & 31 & $3(0$ to 7$)$ & 6 (1 to 11$)$ & 31 (20 to 42$)$ \\
\hline Mental illness & 25 & 3 (0 to 6) & $5(0$ to 10$)$ & 25 (15 to 35$)$ \\
\hline Thoracic medicine & 24 & $2(0$ to 6$)$ & $5(0$ to 10$)$ & 24 (14 to 34$)$ \\
\hline Neurology & 23 & 2 (0 to 6) & 5 (0 to 9 ) & 23 (13 to 33 ) \\
\hline All specialties & 1650 & $165(139$ to 191$)$ & 330 (294 to 366$)$ & $1650(1577$ to 1723$)$ \\
\hline
\end{tabular}

selves to the doctor, or at least a fixed randomly selected proportion of them, and further that the doctor is able to detect and refer them when they arrive, then the referral process can be modelled with the Poisson distribution. This model would describe the minimum variation that might be expected even if all other known factors were taken into account.

A referral rate (L) per person per year, may be calculated from available national or regional data by dividing new referrals during the year $(\mathrm{N})$ for a single specialty or group of specialties ( $\mathrm{s}$ ) by the population being served $(Q)$ (national or regional catchment population for the specialty):

$$
\mathrm{L}_{\mathrm{s}}=\mathrm{N}_{\mathrm{s}} / \mathrm{Q}_{\mathrm{s}}
$$

If the population being served by a doctor is also known $\left(Q_{g p}\right)$ then the number of referrals $(R)$ expected in a given number of years, or parts of a year $(\breve{T})$ is given by the equation:

$$
R_{s}=L_{s} Q_{g p} \breve{T}
$$

The exact probability $(P)$ of a given number of referrals (n) is given by:

$$
P_{n}=R^{n} e^{-R} / n \text { ! }
$$

where $e$ is the natural logarithm base and $n$ ! is factorial $\mathrm{n}(1 \times 2 \times 3 \times 4 \ldots \times \mathrm{n})$.

We can therefore calculate a range within which the actual number of referrals should be found $95 \%$ of the time after taking into account all the other known causes of variation. This is akin to a $95 \%$ confidence interval for the expected number of referrals given the population a doctor serves, the period under consideration, and the specialty. "Consultations" may be substituted as a denominator instead of population being served $\left(\mathrm{Q}_{\mathrm{gp}}\right)$. The same model may be used to estimate the variance expected in various studies given the assumptions in the model. As the number of referrals increases so the normal distribution can be used to provide approximations to the Poisson distribution.

confidence intervals for given numbers of referrals generated by model showing range within which $95 \%$ of time general practitioners would fall

\begin{tabular}{cr}
\hline $\begin{array}{c}\text { Expected } \\
\text { number }\end{array}$ & $\begin{array}{c}\text { 95\% Confidence } \\
\text { interval }\end{array}$ \\
\hline 1 & 0 to 3 \\
5 & 1 to 10 \\
10 & 3 to 17 \\
20 & 11 to 29 \\
30 & 19 to 41 \\
50 & 36 to 64 \\
75 & 58 to 92 \\
100 & 80 to 120 \\
125 & 103 to 147 \\
150 & 125 to 174 \\
200 & 172 to 228 \\
250 & 218 to 281 \\
300 & 266 to 334 \\
400 & 360 to 440 \\
500 & 456 to 544 \\
\hline
\end{tabular}
and 10000 . The figures in brackets give the range in which $95 \%$ of doctors should be found given that the Poisson model is valid. In this type of model the confidence interval is related to the expected number of referrals and not the time or population served. Table II shows a selection of confidence intervals for given numbers of referrals.

The confidence interval for the referral rate is proportional to the confidence interval for the number of referrals. Table I gives some examples. The average general practitioner has around 2000 patients and can be expected to refer seven a week or 330 a year. If the model is valid we would expect the yearly total to fall in the range 294 to 366 on 19 out of 20 occasions ( $95 \%$ ) or 1470 to $1830 / 10000 /$ year. For the most often used specialty, general surgery, the average number of referrals in a year would be 42 with a $95 \%$ confidence interval of 29 to 50 for an average general practitioner (145 to $250 / 10000 / y e a r$ ) or for all specialties over one month 17 to 38 (1020 to $2280 / 10000$ year). For specialties with fewer referrals or individual diagnoses the confidence interval is even greater-for example, in paediatrics an average general practitioner will refer 6.4 children in a year with a $95 \%$ confidence interval of 1 to 12 (5 to 60/10000/year). The same 12-fold variation would be expected in general medicine over four months. Over 20 working days an average general practitioner would refer 18 people with a $95 \%$ confidence interval of 9 to 27 , a threefold variation.

With this model we can simulate studies that have been performed so that extreme ranges can be estimated and compared with the published extreme ranges.

\section{Discussion}

This type of model may be used to explore the impact that random variation may have in the wide variation found in referral rates. Studies give ample examples of considerable differences among individual general practitioners and practices, which are based on sufficient numbers of referrals, so that "random" variations cannot be the cause. The national morbidity studies are possibly the best of these; and they measured practice based referrals over 52 weeks. ${ }^{89}$ Crombie and Fleming have re-examined these studies concentrating on the consistency of referral rates for practices that took part in more than one study. ${ }^{5}$ These practices showed remarkably consistent referral rates when compared over 10 years. The range of referral rates in the third national morbidity survey, based on 60 practices, was six to 55 per 1000 consultations, with a twofold difference between the 20 th and 80 th centiles. Nearly all of this variation must be due to systematic differences in referral behaviour and population characteristics, rather than random variations.

Many studies have examined referral rates for individual practitioners. They are not easy to compare because of the differing definitions used. The Manchester study was one of the largest, in which there were 89030 consultations with 201 general practitioners, resulting in 5467 referrals, a mean referral rate of 6.14 referrals per 100 consultations with a standard deviation of $3 \cdot 16^{23}$ The average number of referrals per general practitioner was $27 \cdot 2$ over the 20 days sampled. With our model and by assuming that each doctor had the same number of consultations on an equivalent population we would have expected $95 \%$ of doctors to have referred between 15 and 41 patients by chance alone, a range of referral rate of 3.39 to 9.26 per 100 consultations. The variance explained by this model could account for at least $15 \%$ of the total found in that study. The model cannot account for the extremes of numbers referred in this study, one to 24 per 100 consultations.

The Manchester study showed a positively skewed distribution of referral rates. Based on the Poisson model we used random numbers to simulate the study 10000 times to examine the distribution of the extreme upper and lower ranges, the highest and lowest number 
of referrals that would be found by chance with 201 "identical" general practitioners. The lower limit of the extreme range for the 201 doctors would fall within the range of 10 to 17 referrals with $95 \%$ confidence. This represents referral rates of $2 \cdot 26$ to 3.84 per 100 consultations. Equivalent figures for the doctor who referred the highest number of patients by random chance are 39 to 49 cases, representing an extreme upper limit of referral rate in the range 8.80 to 11.06 per 100 consultations. The model will thus describe up to a fourfold difference between the highest and lowest referral rates based on random variation but not the 20fold variation that was found, still leaving a substantial part of the variance unexplained.

Dowie's sociological analysis of referral behaviour has been valuable as a basis for building models of behaviour of general practitioners, ${ }^{10}$ yet it was based on referrals to one specialty over three months. She found an average number of 4.9 referrals among 65 doctors, with a standard deviation of 3.5 and extreme range of 0 to 15. The expected confidence intervals for this type of study are wide, with between 0 and 10 referrals expected for a general practitioner with an average list size. The lower end of the extreme range would probably have been 0 to 1 , whereas the upper end of the extreme range had a $95 \%$ confidence interval of 8 to 13 referrals. Random variation accounts for over $40 \%$ of the total variance within this study. Most doctors in the top end of the range were there by chance rather than because of systematically higher referral rates. The figure compares the predicted distribution from our model with Dowie's data.

A much narrower range of referral rates has been found when looking at practices over a sufficient length of time within the Oxford region, in which a threefold variation was measured from the Oxford community health project (A Noone, unpublished data). When these data were standardised for age and sex the practice based referral rates altered only slightly. These data were based on 90 doctors in 33 practices collecting data over 22 weeks, with around 5000 referrals in all. When this study was simulated 10000 times the model predicted that a twofold difference between the highest and lowest referring practices would be found on $13 \%$ of occasions and a less than $1 \cdot 5$-fold difference in the remainder.

These examples show that data based on short periods of observation can lead to a misleading impression of a wide variation in referral rates. Nevertheless, as larger studies show, appreciable variation exists in referral rates between general practitioners. A major step towards exploring further the interface between general practitioners and hospitals is the recent funding of a prototype information system on referrals in East Anglia. Such a regionwide information system is being planned by the performance review unit, a body of general practitioners that is part of the postgraduate medical education structure of the University of Cambridge, and the East Anglian Regional Health Authority. Within two years this system will be giving regular feedback on referrals to East Anglian general practitioners. One of the key issues that has arisen in its development is the assurance that the information can be used to improve the care of patients. General practitioners are unlikely to take part in a practice based audit of their referral behaviour if the information supplied to them on referral rates merely highlights differences that are due to random variation.

There arises a conflict between the perceived needs for presentation of data and the "small numbers" problem discussed. To audit a practice individual cases must be examined. General practitioners had hoped that rates specific to condition and specialty would be identified to highlight those that would warrant further investigation. The model shows that for most specialties and almost all conditions the random variations expected will mask real systematic differences unless long periods are used (in many cases measured in years) or individual patients and doctors are pooled in practices to obtain larger numbers. The second point is likely to mask real differences in individual practice that result from specialisation within the group practice. Rates specific for specialty and diagnosis are particularly prone to this problem as far as interpreting individuals' rates are concerned." It should, however, be possible to use the pooled data to help in determining which specialties and problems show signs that the distributions cannot be accounted for by chance alone and thus help to focus both continuing education and research, including developing ways to use the available resources more effectively. ${ }^{12}$ The same techniques may also be used to measure changes in practice that result from using information to help in directing continuing education programmes. Using the data to monitor individual doctors' responses to information and continuing education at the level of diagnosis or specialty will not be easy.

1 Secretaries of State for Social Services, Wales, Northern Ireland, and Scotland Promoting better health. The government's programme for improving primary health care. London: HMSO, 1987. (Cmnd 249.)

2 Wilkin D, Smith T. Variation in GP referrals to consultants. A report prepared for the DHSS. Manchester: Centre for Primary Care Research, University of Manchester, 1986.

3 Wilkin D Smith A. Explaining variation in general practitioner referrals to hospital. Fam Pract 1987;4:160-9.

4 Secretaries of State for Health, Wales, Northern Ireland, and Scotland. Working for patients. London: HMSO, 1989. (Cmnd 555.)

5 Crombie DL, Fleming DM. General practitioner referrals to hospital: the financial implications of variability. Health Trends 1988;20:53-6.
fint

6 Armitage P. Statistical methods in medical research. Oxford: Blackwell, 1971

7 GP Referral Study Group of the East Anglian Performance Review Unit. Developing a GP referral information system. Report on phase I. Cambridge: GP Performance Review Unit, University of Cambridge, 1988.

8 Royal College of General Practitioners, Office of Population Censuses and Surveys, Department of Health and Social Security. Morbidity statistics from general practice 1971-2. Second national survey. London: HMSO, 1979. (Studies on medical and population subjects, No 36. .)

9 Royal College of General Practitioners, Office of Population Censuses and Surveys, Department of Health and Social Security. Morbidity statistics from general practice 1981-2. Third national study. London: HMSO, 1986. (Series MB5, No 1.)

10 Dowie R. General practitioners and consultants - a study of outpatient referrals. London: Kings Edward's Hospital Fund for London, 1983.

11 Roland M. General practitioner referral rates. Br Med f 1988;297:437-8.

12 Marinker M, Wilkin D, Metcalfe DH. Referrạ! to hospital: can we do better? BrMed f 1988;297:461-4.

(Accepted 2 February 1989)

\section{ANY QUESTIONS}

What is the importance of ventricular bigeminy associated with tachycardia in an elderly patient who is asymptomatic, and does this condition need any antiarrhythmic treatment?

Ventricular bigeminy is not a specific description of a heart rhythm, but it implies that each sinus beat is followed by one ventricular extrasystole in a repetitive fashion. If a person is not only asymptomatic but also free of manifest heart disease, and if the extrasystoles have only one configuration, the condition is of little or no clinical importance. Frequent extrasystoles may, however, be a marker of risk in a patient who does have heart disease, though the association between rhythm and risk may not be directly causal. There is no good evidence that antiarrhythmic treatment solely to suppress extrasystoles in patients who are asymptomatic is useful; such treatment is not recommended whatever the circumstances. I do not know the nature of the tachycardia mentioned by the questioner. An underlying sinus tachycardia may be due to heart failure, thyrotoxicosis, or some other cardiac or systemic disorder. Arrhythmic tachycardias in the elderly usually cause symptoms, although these may not be overtly cardiac. In either case the tachycardia has a better claim on treatment than the extrasystoles. - D A CHAMBERLAIN, consultant cardiologist, Brighton 